Pol. Con. (Edición núm. 26) Vol. 3, No 10

Octubre 2018, pp. 439-450

ISSN: 2550 - 682X

DOI: $10.23857 /$ pc.v3i10.766

Recepción: 08 / 06 / 2018

Aceptación: 23 / 08 / 2018

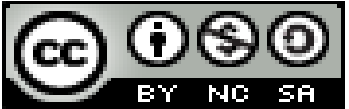

Publicación: 05 / 10 / 2018

Ciencias de salud Artículo de investigación

\title{
Reporte de caso: Lesión duodenal secundaria a traumatismo
}

Case report: Duodenal injury secondary to trauma

\section{Relato de caso: lesão duodenal secundária a trauma}

\author{
Wilson P. Morales-Rosero I \\ mwilsonp@yahoo.com
}

Diego P. Manosalvas-Borjas II

dpmb_msf@hotmail.com

\section{Correspondencia: mwilsonp@yahoo.com}

\footnotetext{
I Doctor en Medicina y Cirugía, Hospital José María Velasco Ibarra Escuela, Superior Politécnica de Chimborazo, Riobamba, Ecuador.

II Medico, Hospital José María Velasco Ibarra, Napo, Ecuador.
} 


\section{Resumen}

La lesión duodenal, debido a su localización retroperitoneal, constituye un gran reto para el diagnóstico médico, gracias a su localización esta suele ser identificada de forma tardía y por consiguiente ha estado asociada a un incremento considerable de la morbimortalidad. Además, debido a su relación anatómica con las demás estructuras abdominales, esta lesione raramente se presenta de forma aislada, como se observó en el presente caso clínico Objetivo. Analizar la correlación existente entre el diagnóstico y terapéutica instaurada en la lesión duodenal secundaria a traumatismo con la evolución en el caso clínico descrito, además de las posibles complicaciones que se pudieran desarrollar. Metodología. Se recopilaron artículos recientes en bases de datos como Pubmed, Elsevier, Springer, New England Journal of Medicine, bajo el criterio de búsqueda de traumatismo abdominal y lesión duodenal excluyéndose aquellos estudios que no fueron concluyentes. Resultados. La lesione duodenal constituye una emergencia médica de gran atención, debido a que la instauración de un tratamiento adecuado no siempre es oportuno, esto por la gran dificultad diagnóstica y las múltiples lesiones que se pueden presentar asociadas en la misma clínica en tal motivo se desarrollan diversas complicaciones que pueden llevar al paciente a la muerte, se describió principalmente al shock hipovolémico que se produce de forma aguda o a la sepsis que se da en etapas crónicas, como principales causas Conclusión. Una vez establecida la necesidad de intervención quirúrgica, debe evaluarse cuidadosamente la extensión de la lesión duodenal y su relación con otras estructuras con el fin de aplicar una terapéutica adecuada.

Palabras clave: lesión duodenal; trauma cerrado de abdomen; fallo multiorgánico; hipovolemia; sepsis.

\section{Abstract}

The duodenal lesion, due to its retroperitoneal location, constitutes a great challenge for the medical diagnosis, thanks to its location this is usually identified late and therefore has been associated with a considerable increase in morbidity and mortality. In addition, due to its anatomical relationship with other abdominal structures, this lesion rarely occurs in isolation, as was observed in the present clinical case Objective. To analyze the existing correlation between the diagnosis and therapy established in the duodenal injury secondary to trauma with the 
evolution in the clinical case described, in addition to the possible complications that could develop. Methodology. We collected recent articles in databases such as Pubmed, Elsevier, Springer, New England Journal of Medicine, under the search criteria for abdominal trauma and duodenal injury, excluding those studies that were inconclusive. Results The duodenal lesion constitutes a medical emergency of great attention, because the establishment of an adequate treatment is not always timely, this due to the great diagnostic difficulty and the multiple injuries that may occur associated in the same clinic in such a case, various complications that can lead the patient to death, was described mainly to hypovolemic shock that occurs acutely or to sepsis that occurs in chronic stages, as the main causes Conclusion. Once the need for surgical intervention is established, the extent of the duodenal lesion and its relationship with other structures should be carefully evaluated in order to apply an adequate therapeutic.

Key words: duodenal injury; closed abdominal trauma; multi-organ failure; hypovolemia; sepsis.

\section{Resumo}

Lesão duodenal, devido à sua localização retroperitoneal, é um grande desafio para o diagnóstico médico, graças à sua localização é normalmente identificado tardiamente e, portanto, tem sido associada com um aumento significativo da morbidade e mortalidade. Além disso, devido à sua relação anatômica com outras estruturas abdominais, essa lesão raramente ocorre isoladamente, como observado no presente caso clínico. Analisar a correlação existente entre o diagnóstico e a terapia estabelecida na lesão duodenal secundária ao trauma com a evolução no caso clínico descrito, além das possíveis complicações que poderiam se desenvolver. Metodologia Artigos recentes são coletadas em bases de dados, tais como Pubmed, Elsevier, Springer, New England Journal of Medicine, sob os critérios de pesquisa de trauma abdominal e lesão duodenal excluindo esses estudos não foram conclusivos. Resultados Duodenal fere constitui uma atenção emergência médica, porque o estabelecimento de um tratamento adequado nem sempre é apropriado, este grande dificuldade diagnóstica e múltiplas lesões que podem ocorrer associada na mesma clínica que razão desenvolver várias complicações que podem levar o paciente à morte, foram descritas principalmente ao choque hipovolêmico que ocorre de forma aguda ou à sepse que ocorre em estágios crônicos, como principais causas Conclusão. Uma vez estabelecida 
a necessidade de intervenção cirúrgica, a extensão da lesão duodenal e sua relação com outras estruturas devem ser cuidadosamente avaliadas, a fim de se aplicar uma terapêutica adequada.

Palavras chave: lesão duodenal; trauma abdominal fechado; falha de múltiplos órgãos; hipovolemia; sépsis.

\section{Introducción}

La lesión duodenal al poseer una localización retroperitoneal constituye un reto diagnóstico para el médico, debido a esta razón en muchas ocasiones es identificada de manera tardía y se asocian a un aumento de las complicaciones y de la mortalidad de los pacientes. Los avances en el diagnóstico precoz de esta entidad, así como la evolución de las estrategias para el manejo médico ha dado lugar a disminución en las cifras en la mortalidad con respecto a las series iniciales del manejo quirúrgico de la misma en las cuales se informaban una mortalidad del $100 \%$, sin embargo a día de hoy, la mortalidad se ha logrado reducir a un promedio de y se mantiene entre un 4,5 y 30\%, según estadísticas de García et Al y una relación 5:a de hombres a mujeres, en la actualidad esta tasa de mortalidad se ha mantenido estancada, lo que demuestra la compleja naturaleza de estos siendo la causa de muerte aguda al shock hipovolémico y la tardía producida por sepsis secundaria.

\section{Caso Clínico}

Paciente masculino de 23 años, el cual inicia su cuadro clínico actual hace 45 minutos previo a su ingreso, sufre lesión por aplastamiento a nivel abdominal por neumático de vehículo pesado (camión) secundario a impacto de motocicleta de la cual es eyectado, lo que produce múltiples lesiones excoriativas en región facial, abdominal y extremidades. Al momento de su ingreso fue manejado bajo esquema ATLS de trauma. Signos vitales: frecuencia cardiaca 87 latidos por minuto, frecuencia respiratoria 21 respiraciones por minuto, tensión arterial 74/41 mmHg, temperatura $36,4^{\circ} \mathrm{C}$, saturación 97\%. Examen físico: Paciente desorientado con signos de intoxicación alcohólica, abdomen tenso con varias escoriaciones, la mayor de ellas en fosa iliaca derecha, abdomen tenso, Bloomberg positivo, adicional a esto se aprecia herida cortante de aproximadamente $3 \mathrm{~cm}$ en cara anterior de pierna derecha, que compromete piel 
Biometría hemática: Leucocitos $18700 \mathrm{~mm} 3$, neutrófilos 60,6\%, linfocitos 32\%, hemoglobina $14,1 \mathrm{~g} / \mathrm{dL}$, Hematocrito $42 \%$,

\section{FAST: Positivo en Morrison.}

Laparotomía exploratoria: Hemoperitoneo de aproximadamente 2500ml, laceración de arteria cólica media con sangrado activo, ruptura de vasos colaterales en colon transverso, laceración de meso a nivel de colon transverso, perforación de aproximadamente $2 \mathrm{~cm}$ con salida de heces fecales en colon transverso, adenopatías mesentéricas, hematoma no expansivo en fondo y fosa ilíaca derecha.

Se realiza evacuación de hemoperitoneo, colocación de puntos simples en arteria cólica media, ligadura de arterias segmentarias en mesocolon, rafia colónica en 2 planos más cierre, secado de cavidad más visualización de órganos, apendicetomía convencional, colocación de dren a nivel de rafia.

Evolución: 48 horas posteriores a primera laparotomía, paciente álgico, frecuencia cardiaca 108 latidos por minuto, frecuencia respiratoria 24 respiraciones por minuto facies pálidas, abdomen tenso y doloroso, producción de dren total en 24 horas de $1150 \mathrm{ml}$ de líquido hemático.

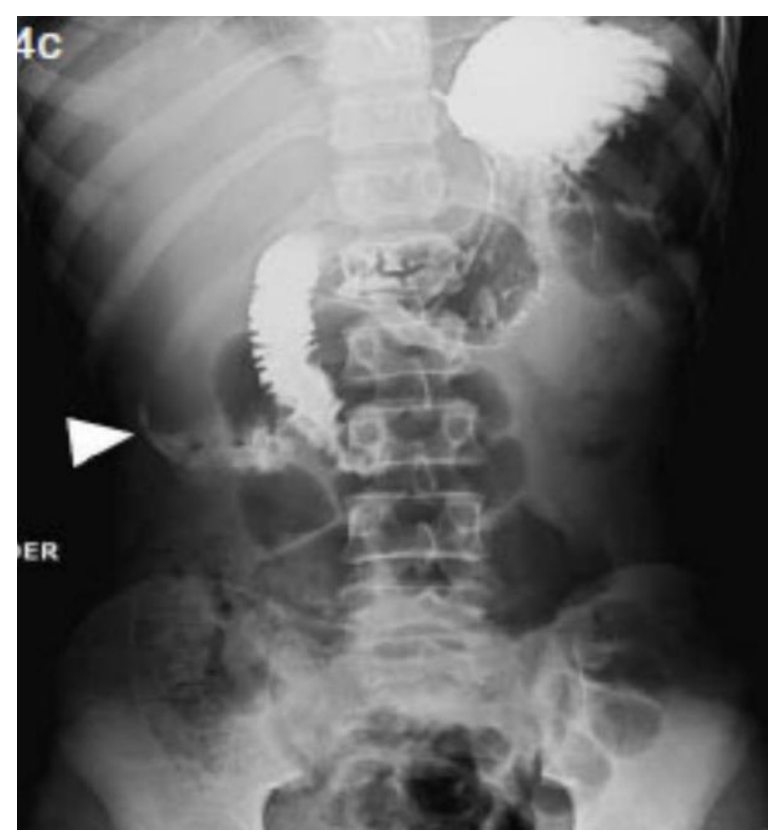

IMAGEN 1 
Radiografía de abdomen con contraste oral. Se confirma perforación duodenal observándose extravasación de contraste a nivel de la segunda porción del duodeno (flecha blanca)

Segunda laparotomía exploratoria: perforación de aproximadamente $4 \mathrm{~cm}$ de diámetro en cara anterior de tercera porción de duodeno, bilioma subhepático de $200 \mathrm{ml}$, rafias previas en raíz de mesenterio. En base a dichos hallazgos se realiza aspiración de bilioma, Maniobra de Kocher ampliada, rafia primaria de lesión duodenal en dos planos. Exclusión duodenal con posterior gastrostomía, yeyunostomía retrógrada, yeyunostomía anterógrada a 60cm del ángulo de Tritz, y piloroplastia.

\section{IMAGEN 2}

Exposición de perforación en cara anterior de tercera porción de duodeno de aproximadamente 4 $\mathrm{cm}$

\section{IMAGEN 3}

Imagen muestra heridas quirúrgicas y colocación de drenajes para manejo del paciente 
Tras 4 días de estancia en sala de cuidados intermedios, con evolución favorable, es remitido a sala general de cirugía hombres, donde permanece por 14 días, y es dado de alta. Al momento en seguimiento por consulta externa.

\section{Discusión}

La mayoría de las lesiones duodenales son producto de traumatismo a nivel abdominal, el diagnóstico inicial se produce generalmente en el momento de la laparotomía exploratoria.1,4,6 La inestabilidad hemodinámica del paciente de trauma abdominal se maneja mejor en la sala de operaciones, omitiendo cualquier evaluación imágenes preoperatorias. 1,11

La contusión duodenal se sospecha si hay engrosamiento de la pared, hematoma intramural de alta densidad, o edema; la presencia de la discontinuidad de la pared, fluido periduodenal, gas extraluminal en el retroperitoneo son hallazgos sugestivos de perforación duodenal.11

La evaluación meticulosa intraoperatoria del duodeno se lleva a cabo mediante la realización de una rotación visceral medial derecha de la flexura cólica derecha y una maniobra de Kocher para proporcionar la exposición de las segunda y tercera porciones del duodeno y la cabeza del páncreas; para visualizar mejor la cuarta porción del duodeno, y para una evaluación completa del páncreas, se debe bajar el ligamento gastrocólico y el omento menor. Además de esto el ligamento de Treitz se puede movilizar para exponer el duodeno distal durante la rotación visceral medial.3, 12 es este protocolo descrito por la literatura el que se encuentra poco claro en el protocolo de la primera intervención y que es descrito en la reintervención del paciente

Es necesaria una gran cantidad de energía para causar lesión duodenal contundente, la mayoría de estos pacientes llegan al hospital normotensos, sin alteración significativa en el estado de consciencia, y carecen de pruebas externas de traumatismo abdominal, tales como equimosis o abrasiones; los signos característicos en esta entidad y en estos casos son el dolor en hipocondrio y flanco derechos asociado a distensión abdominal, sin embargo estos signos no son específicos de esta entidad, lo que dificulta su diagnóstico.4,6,8

La peritonitis con inestabilidad hemodinámica requiere exploración quirúrgica3,4 en los casos que no exista dicha inestabilidad los estudios de imagen representan un excelente apoyo diagnóstico 
La Tomografía Computarizada Abdominal tiene una sensibilidad diagnóstica del 84-100\% en la población de pacientes con trauma abdominal cerrado por lesiones de víscera hueca; atendiendo a este estudio se puede clasificar a la lesión duodenal de acuerdo al criterio de varios autores, muchos plantean que el diagnóstico debe hacerse con la identificación de cualquier cantidad de fluidos en cavidad abdominal, otros estudios plantean que para el diagnóstico se requieren de 3 mm o más de engrosamiento de la pared intestinal, aire libre, extravasación de contraste, o cantidades más grandes de fluido libre en la cavidad abdominal.2,5

En pacientes en los que la TC abdominal no arroja resultados concluyentes y la sintomatología que presentan aun concuerda con traumatismo asociado a perforación de víscera hueca es de muy poca ayuda el llevar a cabo otro examen preoperatorio. En estos pacientes, la exploración abdominal, así como la planificación de TC abdominal en cierto intervalo de tiempo puede ser una opción, siempre y cuando el paciente se encuentre en condiciones estables, La laparoscopia diagnóstica tiene un papel cada vez mayor en la evaluación del trauma cerrado de abdomen.

Otro medio diagnóstico es el examen FAST por ultrasonido, empleado para la evaluación principalmente del cuadrante superior derecho, este evalúa el espacio hepatorenal, la bolsa de Morrison y la base pulmonar derecha; además es el más sensible para el diagnóstico de hemoperitoneo y el más fácil de obtener en el sentido técnico. Es muy importante la evaluación del diafragma para determinar la presencia de un hemotórax potencial.11,12

\section{Clasificación}

La lesión duodenal puede clasificarse en grados I a V en base a la presencia de hematoma o laceración y el grado de implicación de la circunferencia duodenal.10,11

\section{Grado 1:}

Hematoma que ocupa una sola porción del duodeno; con laceración parcial sin perforación.

\section{Grado 2:}

Hematoma que involucra más de una porción duodenal; con laceración de menos del 50\% de la circunferencia duodenal. 


\section{Grado 3:}

Interrupción de la circunferencia $50-75 \%$ de la segunda porción, o interrupción $50-100 \%$ de la primera, tercera o cuarta porciones.

\section{Grado 4:}

Interrupción de $>75 \%$ de circunferencia de segunda parte, involucrando ampolla o distal conducto biliar común.

\section{Grado 5:}

Alteración masiva del complejo duodenopancreático, con devascularización del duodeno.

El manejo dependerá del grado en el que se clasifique al paciente; así pues:

Para los hematomas intramurales de grado I y II diagnosticados por TC, el manejo inicial consiste en terapia no quirúrgica; muchos de estos pacientes tendrán trastornos gástricos, obstrucción del tránsito intestinal que se acompaña de náuseas, con o sin emesis biliosa; el tratamiento implica descompresión proximal mediante la colocación de una sonda nasogástrica y provisión de nutrición parenteral; con esta estrategia, la mayoría de los hematomas intramurales se resuelven en un promedio de tres semanas.7

El manejo de las lesiones de grado III requiere un título de juicio clínico e individualización de procedimientos quirúrgicos. La mejor opción es la reparación o resección con anastomosis primaria así como implementar procedimientos adicionales para proteger la reparación o limitar las consecuencias del fallo; la determinación inicial es definir si una reparación primaria del duodeno es factible; el cirujano necesita considerar el grado de la circunferencia y la extensión de tejido desvitalizado a la hora de decidir el procedimiento adecuado para la operación, los desgarros de hasta el $75 \%$ de la circunferencia duodenal pueden ser reparado de forma segura dependiendo principalmente en su ubicación, la capacidad de cerrar el defecto sin tensión, y presencia de perfusión adecuada.1,11

Las lesiones de grado IV y V implican una desvascularización significativa del duodeno o alteración de la Ampolla de Vater y complejo duodenopancreático. Los pacientes que presentan este grado de trauma suelen presentar ya sea en shock o con lesiones severas concomitantes. a 
otros órganos, en estos pacientes se debe buscar una estrategia para el control de daños, centrándose en la hemorragia, control y desbridamiento del tejido desvitalizado y uso de drenaje externo en un esfuerzo por minimizar el tiempo operatorio y corrección de la tríada letal.11

La mortalidad en pacientes con lesiones duodenales posee la misma tendencia general de mortalidad que la evidenciada en pacientes que han sufrido traumas mayores, en donde el 73$80 \%$ de las muertes son evidenciadas dentro de las primeras 48 horas y no están relacionados directamente con la lesión duodenal, en su lugar la muerte en estos pacientes está relacionada con hemorragia o lesión cerebral traumática y es atribuible comúnmente a la sepsis y la insuficiencia multiorgánica que se produce.

Se informa que de forma variable del 1 al $4 \%$ en la mayoría de estos casos, la mortalidad se debe a sepsis que se desarrolla a partir de una fístula duodenal.

\section{Complicaciones}

Las lesiones duodenales están relacionadas con un alto índice de complicaciones, estas surgen debido a la formación de fístulas resultantes de un fallo en la reparación quirúrgica por dehiscencia de la línea de sutura y está ocasionalmente representada por la obstrucción duodenal que se produce en estos pacientes.1 Entre las complicaciones que suelen aparecer encontramos:

Absceso intraabdominal (15\%)

Fistula duodenal $(6 \%)$

Obstrucción duodenal $(0,9 \%)$

Pancreatitis recurrente $(0,6 \%)$

\section{Mortalidad}

La mortalidad secundaria a traumatismo con afectación duodenal puede dividirse en temprana y tardía.

Las muertes tempranas son causadas en la mayoría de casos por exanguinación, la cual es resultante de lesiones vasculares mayores. 
Las muertes tardías se deben principalmente a sepsis, formación de fístulas duodenales y fallo multiorgánico. 1,11

\section{Conclusión}

La lesión duodenal es un evento muy poco común cuyo manejo es desafiante debido a la complejidad de realizar un diagnóstico preciso y oportuno, la ubicación anatómica que complica la exposición para la evaluación y la reparación de las lesiones que presente el paciente a nivel intestinal, además de las posibles lesiones a nivel de otros órganos que acompañen a esta entidad y por ultima la falta de experiencia del cirujano debido a la rareza de esta entidad.

A pesar de un gran número de publicaciones, la mayoría de estas están limitadas a una determinada población y sujetas a una evaluación retrospectiva, la cual deja el campo abierto para un estudio adicional del manejo óptimo de este tipo de lesiones complejas.

\section{Referencias Bibliográficas}

García Santos E, Soto Sánchez A, Verde J, Marini C, Asensio J, Petrone P. Lesiones duodenales secundarias a traumatismo: revisión de la literatura. Cirugía Española. 2015;93(2):68-74.

Nima Kokabi, et al. Specific Radiological Findings of Traumatic Gastrointestinal Tract Injuries in Patients with Blunt Chest and Abdominal Trauma. HVI in BAT / Canadian Association of Radiologists Journal 66 (2015) 158e163.

Zavala-Retes B, Ortiz-Barrón S, Guzmán-Valdivia-Gómez G, Guerrero-Aguirre J, Salazar Lozano CR. Manejo quirúrgico de una perforación duodenal tardía asociada con sepsis abdominal secundaria a la ingesta incidental de un mondadientes. Rev Esp Med Quir 2014; $19: 363-370$.

Phillips B, Turco L, McDonald D, Mause A, Walters R. Penetrating injuries to the duodenum. Journal of Trauma and Acute Care Surgery. 2017;83(5):810-817.

Chen C, Schuster K, Bhattacharya B. Motor Vehicle Collision Patient with Simultaneous Duodenal Transection and Thoracic Aorta Injury: A Case Report and Review of the Literature. Case Reports in Surgery. 2015; 2015:1-3. 
Hartholt K, Dekker J. Duodenal perforation as result of blunt abdominal trauma in childhood. BMJ Case Reports. 2015; bcr2015213330.

Prajapati D, Rampal K, Soni M, Prajapati J. Case Report on Two Large Duodenal Perforations and Their Management in Emergency Conditions. International Journal of Integrative Medical Sciences. 2016;3(5):280-284.

Petrone P, Magadán Álvarez C, Joseph D, Cartagena L, Ali F, E.M. Brathwaite C. Abordaje y manejo de las lesiones retroperitoneales traumáticas. Cirugía Española. 2018;96(5):250-259.

Johnston L, Wind G, Bradley M. Duodenal trauma. Trauma. 2017;19(2):94-102.

Giraldo-Restrepo JA, Serna-Jiménez TJ. Examen FAST y FAST extendido. Rev Colomb Anestesiol.2015;43:299-306. 